\title{
Gravitational waves and neutrino emission from the merger of binary neutron stars
}

\author{
Yuichiro Sekiguchi, Kenta Kiuchi, Koutarou Kyutoku, and Masaru Shibata \\ Yukawa Institute for Theoretical Physics, Kyoto University, Kyoto 606-8502, Japan
}

\begin{abstract}
Numerical simulations for the merger of binary neutron stars are performed in full general relativity incorporating a finite-temperature (Shen's) equation of state (EOS) and neutrino cooling for the first time. It is found that for this stiff EOS, a hypermassive neutron star (HMNS) with a long lifetime $(\gg 10 \mathrm{~ms})$ is the outcome for the total mass $\lesssim 3.0 M_{\odot}$. It is shown that the typical total neutrino luminosity of the HMNS is $\sim 3-8 \times 10^{53} \mathrm{erg} / \mathrm{s}$ and the effective amplitude of gravitational waves from the HMNS is $4-6 \times 10^{-22}$ at $f=2.1-2.5 \mathrm{kHz}$ for a source distance of $100 \mathrm{Mpc}$. We also present the neutrino luminosity curve when a black hole is formed for the first time.
\end{abstract}

PACS numbers: 04.25.Dm, 04.30.-w, 04.40.Dg

Introduction: Coalescence of binary neutron stars (BNS) is one of the most promising sources for nextgeneration kilo-meter-size gravitational-wave (GW) detectors [1], and also a possible candidate for the progenitor of short-hard gamma-ray bursts (SGRB) [2]. Motivated by these facts, numerical simulations have been extensively performed for the merger of BNS in the framework of full general relativity in the past decade since the first success in 2000 [3] (see, e.g., [4] for a review).

BNS evolve due to gravitational radiation reaction and eventually merge. Before the merger sets in, each neutron star is cold (i.e., thermal energy of constituent nucleons is much smaller than the Fermi energy), because thermal energy inside the neutron stars is significantly reduced by neutrino and photon coolings due to the long-term evolution (typically $\gtrsim 10^{8}$ yrs) until the merger [5]. By contrast, after the merger, shocks are generated by hydrodynamic interactions. In particular, when a hypermassive neutron star (HMNS) is formed, spiral arms are developed in its envelope and continuous heating occurs due to the collision between the HMNS and spiral arms (e.g., [6 8] ). By this process the maximum temperature increases to $\sim 30-50 \mathrm{MeV}$, and hence, copious neutrinos are emitted 9 11]. To accurately study the evolution of the hot HMNS with a physical modeling, we have to incorporate microphysical processes such as neutrino emission and equation of state (EOS) based on a theory for the high-density and high-temperature nuclear matter. However, such simulations have not been done yet in full general relativity (but see [12] for a work in an approximate general relativistic gravity with finite-temperature EOSs). Incorporation of microphysical processes is in particular important for exploring the merger hypothesis of SGRB because it may be driven through pair annihilation of neutrino-antineutrino pairs [2].

In this letter, we present the first results of numericalrelativity simulation for the BNS merger performed incorporating both a finite-temperature (Shen's) EOS [13] and neutrino cooling [14]. In the following, we report the possible outcome formed after the merger, criterion for the formation of HMNS and black hole $(\mathrm{BH})$, thermal properties of the HMNS, and neutrino luminosity from the HMNS and in the $\mathrm{BH}$ formation.
Setting of numerical simulations: Numerical simulations in full general relativity are performed using the following formulation and numerical schemes. Einstein's evolution equations are solved in the so-called BSSNpuncture formulation [15]. We employ the original version of the geometric variables for the BSSN formulation together with an improved definition of the conformal factor. As in 7], the dynamical gauge condition for the lapse function and shift vector is chosen; a fourthorder-accurate finite differencing in space and a fourthorder Runge-Kutta time integration are used; a conservative shock capturing scheme with third-order accuracy in space and fourth-order accuracy in time is employed for solving hydrodynamic equations. In addition to the ordinary hydrodynamic equations, we solve evolution equations for the neutrino $\left(Y_{\nu}\right)$, electron $\left(Y_{e}\right)$, and total lepton $\left(Y_{l}\right)$ fractions per baryon, and, taking into account weak interaction processes [14]. Shen's EOS, tabulated in terms of the rest-mass density $(\rho)$, temperature $(T)$, and $Y_{e}$ or $Y_{l}$, is employed. In addition, we incorporate neutrino cooling, employing a general relativistic leakage scheme [14]. In our leakage scheme, electron neutrinos $\left(\nu_{e}\right)$, electron antineutrinos $\left(\bar{\nu}_{e}\right)$, and other types $(\mu / \tau)$ of neutrinos $\left(\nu_{x}\right)$ are taken into account.

Numerical simulations are performed preparing a nonuniform grid as in 7]. The inner domain is composed of a finer uniform grid and the outer domain of a coarser nonuniform grid. The grid resolution in the inner zone is chosen so that the major diameter of each neutron star in the inspiral orbit is covered by 60 and 80 grid points for low- and high-resolution runs, respectively: We always performed simulations for both grid resolutions to confirm that convergence is approximately achieved. Outer boundaries are located in a local wave zone (at $\approx 560$ $600 \mathrm{~km}$ along each coordinate axis which is longer than gravitational wavelength in the inspiral phase). During the simulations, we check the conservation of baryon restmass, total gravitational mass (Arnowitt-Deser-Misner mass plus radiated energy of GWs), and total angular momentum (including that radiated by $\mathrm{GWs}$ ), and find that the errors are within $0.5 \%, 1 \%$, and $3 \%$, respectively, for the high-resolution runs within the duration $\approx 30 \mathrm{~ms}$.

Shen's EOS, derived from a relativistic mean-field the- 


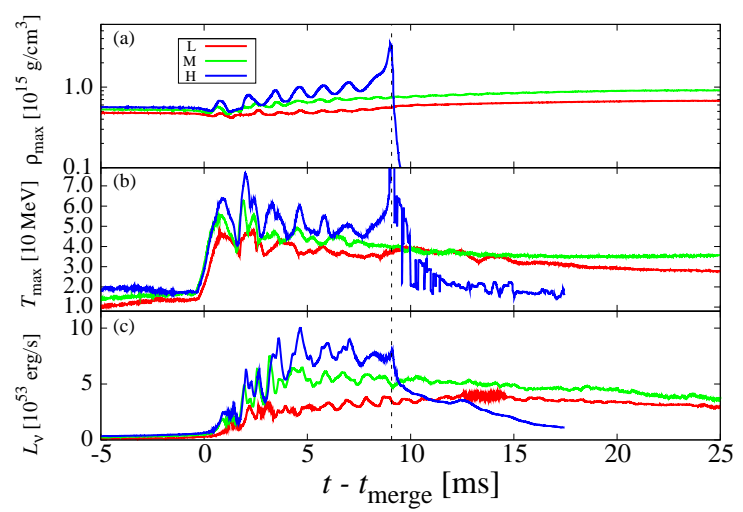

FIG. 1: Maximum rest-mass density, maximum matter temperature, and total neutrino luminosity as functions of time for three models. The dashed vertical line shows the time at which a $\mathrm{BH}$ is formed for model $\mathrm{H}$.

ory [13], is a stiff one among other EOSs, giving the maximum mass of zero-temperature spherical neutron stars $M_{\max } \approx 2.2 M_{\odot}$. The latest discovery of a high-mass neutron star with mass $1.97 \pm 0.04 M_{\odot}$ [16] suggests that stiff EOSs are favored, and Shen's EOS satisfies this requirement. There are two possible fates of BNS [] $]$ : If its total mass $M$ is larger than a critical mass $M_{c}$, a $\mathrm{BH}$ will be formed soon after the onset of the merger, while a differentially rotating HMNS will be formed for $M<M_{c}$. The value of $M_{c}$ depends strongly on the EOS. Because Shen's EOS is quite stiff, $M_{c}$ is much larger than the typical total mass of BNS, $\sim 2.7 M_{\odot}$ [17], as shown below. Thus, with this EOS, a HMNS is the frequent outcome, as in the cases of stiff EOSs with which $M_{\max }>2 M_{\odot}$ [18].

This paper focuses on the merger of equal-mass BNS with three masses for each neutron star: $M_{\mathrm{NS}}=1.35,1.5$, and $1.6 M_{\odot}\left(M_{\mathrm{NS}}\right.$ is the gravitational mass of a neutron star in isolation). We refer to each model as models $\mathrm{L}$ (light), $\mathrm{M}$ (middle), and $\mathrm{H}$ (heavy). We perform the simulation with the initial condition of about 3-4 orbits before the onset of the merger until the system relaxes to a quasistationary state. Quasiequilibrium states of BNS are prepared as the initial conditions, as in [6, 7].

Numerical results: Figure 1 plots the evolution of the maximum rest-mass density, $\rho_{\max }$, maximum matter temperature, $T_{\max }$, and total neutrino luminosity as functions of $t-t_{\text {merge }}$ where $t_{\text {merge }}$ is the onset time of the merger. For $t<t_{\text {merge }}, \rho_{\max }$ is approximately constant, while $t \gtrsim t_{\text {merge }}$, a HMNS is formed and subsequently contracts by emission of GWs, which carry energy and angular momentum from the HMNS; $\rho_{\max }$ increases in the gravitational radiation timescale. However, at $t-t_{\text {merge }} \sim 20 \mathrm{~ms}$ for models $\mathrm{L}$ and $\mathrm{M}$, the degree of its nonaxial symmetry becomes low enough that the emissivity of GWs is significantly reduced. Because no dissipation process except for neutrino cooling is present, the HMNS will be alive at least for the cooling timescale before collapsing to a $\mathrm{BH}$ (see below). For model $\mathrm{H}$, the HMNS eventually collapses to a $\mathrm{BH}$ after the gradual contraction due to the GW emission and a massive disk of $\approx 0.1 M_{\odot}$ is formed around the $\mathrm{BH}$.

The evolution of $T_{\max }$ plotted in Fig. 1(b) shows that HMNS just after the formation are hot with $T_{\max } \sim 50$ $70 \mathrm{MeV}$. Such high temperature is achieved due to the liberation of kinetic energy of the orbital motion at the collision of two neutron stars. Subsequently, $T_{\max }$ decreases due to the neutrino cooling, with the maximum luminosity $3-10 \times 10^{53} \mathrm{erg} / \mathrm{s}$ (see Fig. 1(c)), but relaxes to a high value with $25-50 \mathrm{MeV}$. Around the HMNS, spiral arms are formed and shock heating continuously occurs when the spiral arms hit the HMNS (cf. Fig. 2). Due to this process and because of a long neutrino cooling timescale (see below), the temperature (and thermal energy) does not significantly decrease in $\sim 10-100 \mathrm{~ms}$.

Figure 2 plots the color maps of rest-mass density, matter temperature, and total neutrino luminosity for model $\mathrm{M}$ at $t-t_{\text {merge }}=15 \mathrm{~ms}$, at which it relaxes to a semi-final quasisteady state. This shows that the HMNS is weakly spheroidal and the temperature is high in its outer region. The neutrino luminosity is also high in its outer region, in particular, near the polar surface. With the fact that the rest-mass density is relatively small near the rotation axis above the polar surface, this is a favorable feature for the merger hypothesis of SGRB; pair annihilation of neutrinos and antineutrinos could supply a large amount of thermal energy which may drive a fire ball along the rotation axis. The pair annihilation efficiency has been approximately estimated in the previous works [-11]. These show that the efficiency for $\nu_{e} \bar{\nu}_{e}$ annihilation is $\sim 10^{-2}\left(L_{\nu_{e}} / 10^{53} \mathrm{erg} / \mathrm{s}\right)$. If this result holds in our work, the pair annihilation luminosity would be $\sim 10^{51} \mathrm{erg} / \mathrm{s}$.

The reasons that HMNS are formed are; (i) it is rapidly rotating with the period $\sim 1 \mathrm{~ms}$, and hence, the centrifugal force increases the possible mass that can be sustained; (ii) because it is hot, thermal energy enhances the pressure. We find that the rotational velocity with the period $\sim 1 \mathrm{~ms}$ does not play a substantial role. Exploring in detail Shen's EOS for high density tells us that the effect of the thermal energy is significant and can increase $M_{\max }$ by $\sim 20-30 \%$ for a high-temperature state with $T \gtrsim 20 \mathrm{MeV}$. Therefore, the HMNS will alive before collapsing to a $\mathrm{BH}$ for a long cooling time $E_{\mathrm{th}} / L_{\nu} \sim 2-3 \mathrm{~s}$ where $E_{\text {th }}$ is total thermal energy of the HMNS. At the time when the HMNS collapses to a $\mathrm{BH}$, it will be close to a spherical configuration with low temperature due to longterm GW and neutrino emissions. Thus, observable signals from the late-time collapse will be weak.

Figure 3 plots neutrino luminosities as functions of time for three flavors $\left(\nu_{e}, \bar{\nu}_{e}\right.$, and sum of $\left.\nu_{x}\right)$. It is found that electron antineutrinos are dominantly emitted for any model. The reason for this is as follows: The HMNS has a high temperature, and hence, electron-positron pairs are efficiently produced from thermal photons, in particular in its envelope. Neutrons efficiently capture the positrons to emit antineutrinos whereas electrons are not captured by protons as frequently as positrons because the proton fraction is much smaller. Such hierarchy 


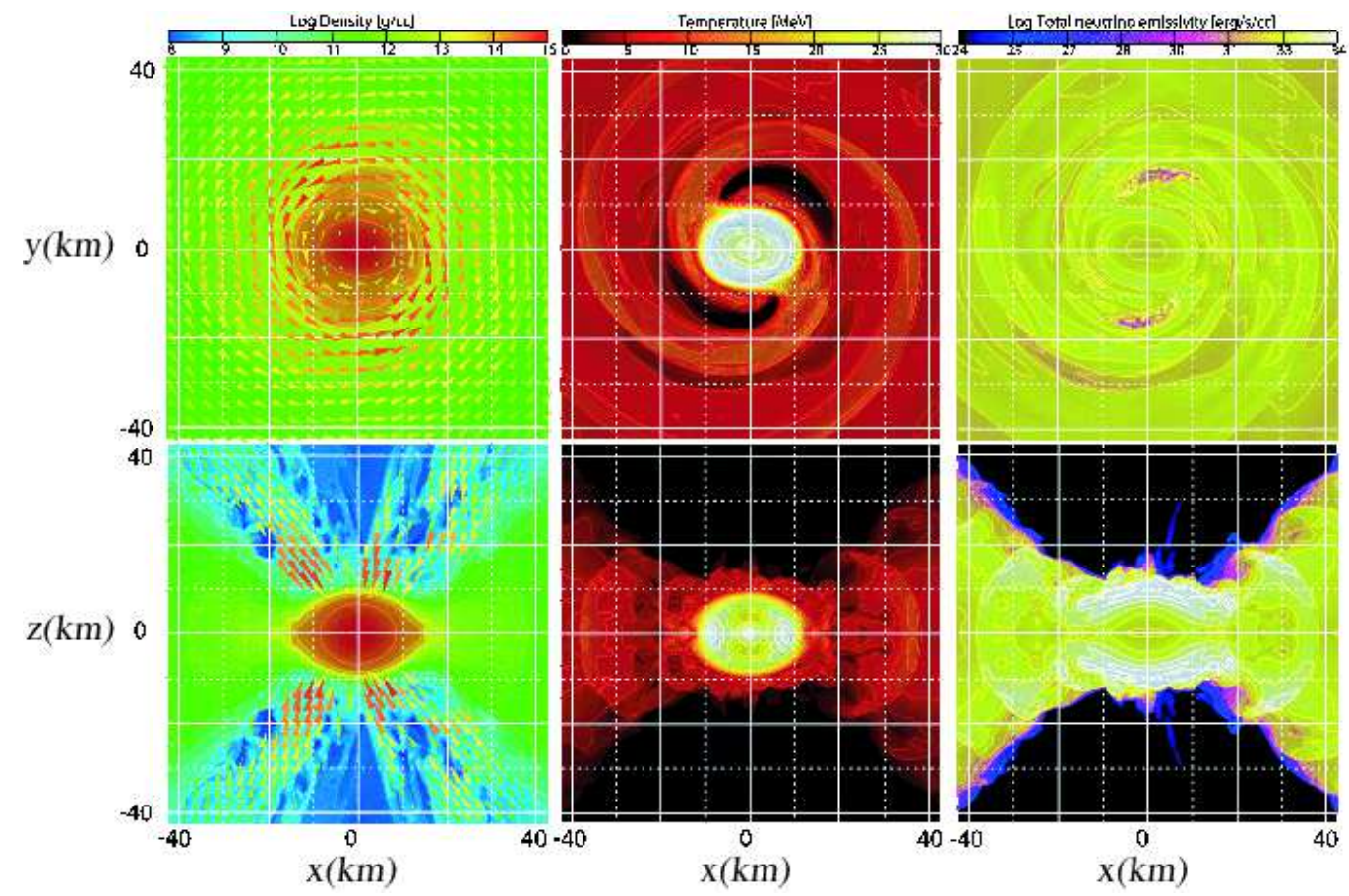

FIG. 2: Color maps of rest-mass density (with velocity fields), temperature, and total neutrino luminosity at $t \approx 15 \mathrm{~ms}$ after the merger for model M. The upper and lower panels show the configuration in the $x-y$ and $x$ - $z$ planes, respectively.

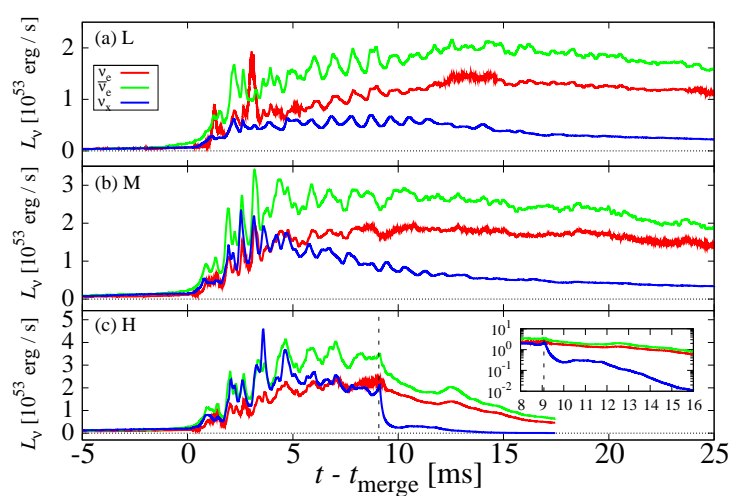

FIG. 3: Neutrino luminosities for three flavors for three models. The inset of the bottom panel focuses on the luminosities in the $\mathrm{BH}$ formation. The meaning of the dashed line is the same as in Fig. 1 .

in the neutrino luminosities was reported also in [9].

Soon after the $\mathrm{BH}$ formation for model $\mathrm{H}, \mu / \tau$ neutrino luminosity steeply decreases because high temperature regions are swallowed into the $\mathrm{BH}$, while luminosities of electron neutrinos and antineutrinos decrease only gradually because these neutrinos are emitted via chargedcurrent processes from the massive accretion disk. We here note that magnetic fields, which are not taken into account in the present simulations, could be amplified significantly in the accretion disk [8] and may play a role in the late evolution of the BH-disk system.

The antineutrino luminosity for the long-lived HMNS is $L_{\bar{\nu}} \sim 1.5-3 \times 10^{53} \mathrm{erg} / \mathrm{s}$ with small time variability. It is by a factor of $\sim 1-5$ larger than that from protoneutron stars formed after supernovae 19]. Averaged neutrino energy is $\epsilon_{\bar{\nu}} \sim 20-30 \mathrm{MeV}$. The sensitivity of water-Cherenkov neutrino detectors such as SuperKamiokande and future Hyper-Kamiokande (HK) have a good sensitivity for such high-energy neutrinos in particular for electron antineutrinos 20]. The detection number for electron antineutrinos is approximately estimated by $\sigma \Delta T L_{\bar{\nu}} /\left(4 \pi D^{2} \epsilon_{\bar{\nu}}\right)$ where $\sigma$ is the total cross section of the detector against target neutrinos, $\Delta T$ is the lifetime of the HMNS, and $D$ is the distance to the HMNS. For a one-Mton detector such as HK, the expected detection number is $\gtrsim 10$ for $D \lesssim 5 \mathrm{Mpc}$ with $\Delta T \sim 2-3 \mathrm{~s}$, based on an analysis of [20]. Thus, if the BNS merger fortunately happens within $D \sim 5 \mathrm{Mpc}$, neutrinos from the HMNS may be detected and its formation may be confirmed. Note that GWs from the HMNS will be simultaneously detected for such a close event (see below), reinforcing the confirmation of the HMNS formation.

Figure 4 (a) plots gravitational waveforms as a function of retarded time $t_{\text {ret }}=t-D-2 M \log (D / M)$ for three models where $M=2 M_{\mathrm{NS}}$. Here, $h_{+}$and $h_{\times}$denote the plus and cross modes of GWs extracted from the metric in the local wave zone. The waveforms are composed of the so-called chirp waveform, which is emitted when the BNS is in an inspiral motion (for $t_{\text {ret }} \lesssim t_{\text {merge }}$ ), and the merger waveform (for $t_{\text {ret }} \gtrsim t_{\text {merge }}$ ), on which we here focus. For the HMNS formation, the merger waveforms are composed of quasiperiodic waves for which $h \lesssim 10^{-22}$ for $D=100 \mathrm{Mpc}$ and the peak frequencies are in a narrow range $f_{\text {peak }}=2.1-2.5 \mathrm{kHz}$ depending weakly on $M$. They agree with that in the approximate general relativistic study [12]. Note that $f_{\text {peak }}$ depends on adopted 

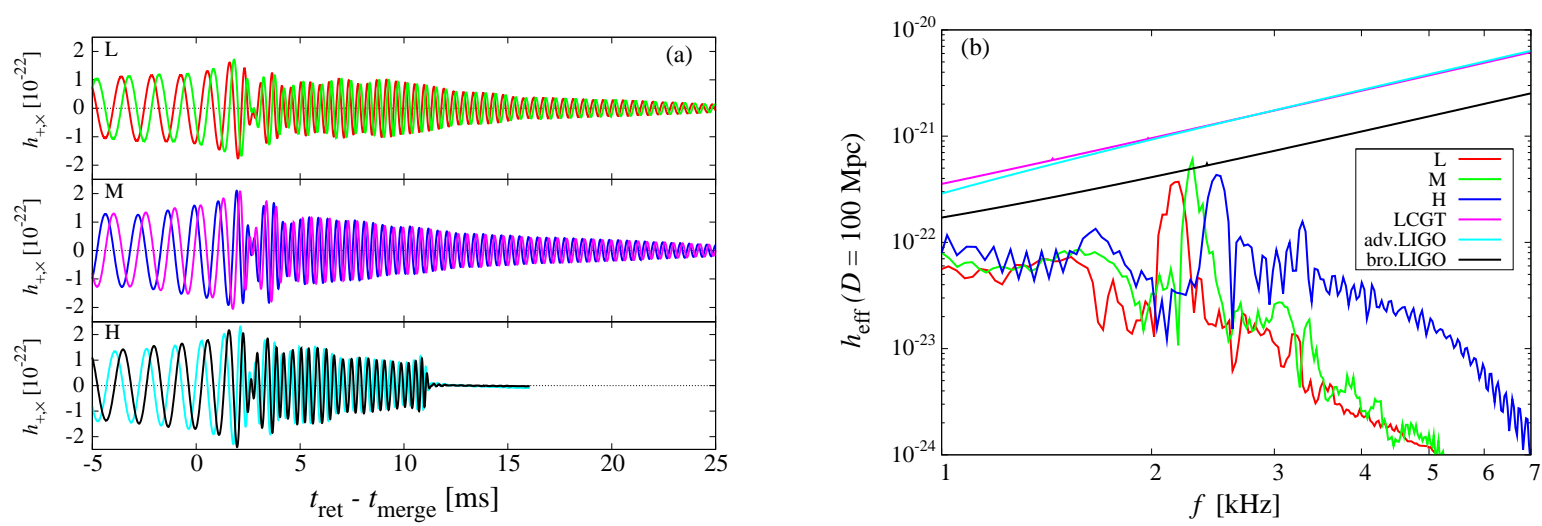

FIG. 4: (a) GWs observed along the axis perpendicular to the orbital plane for the hypothetical distance to the source $D=100 \mathrm{Mpc}$. (b) The effective amplitude of GWs as a function of frequency for $D=100$ Mpc. The noise amplitudes of Advanced Laser Interferometer Gravitational wave Observatories (adv. LIGO), broadband configuration of Advanced LIGO (bro. LIGO), and Large-scale Cryogenic Gravitational wave Telescope (LCGT) are shown together.

EOS [12], and we will describe the dependence of GWs on EOS elsewhere. The accumulated effective amplitude, $h_{\mathrm{eff}} \equiv 0.4 h(f \Delta T)^{1 / 2}$, is much larger where the factor 0.4 comes from the averages of angular direction of the source and rotational axis of the HMNS. Figure 4(b) shows the effective amplitude defined by $0.4 h(f) f \sim 4-6 \times 10^{-22}$ for $D=100 \mathrm{Mpc}$, where $h(f)$ is the absolute value of the Fourier transformation of $h_{+}+i h_{\times}$. This suggests that for a specially-designed version of advanced GW detectors such as broadband LIGO, which has a good sensitivity for a high-frequency band, GWs from the HMNS oscillations may be detected with $\mathrm{S} / \mathrm{N}=5$ if $D \lesssim 20 \mathrm{Mpc}$ or the source is located in an optimistic direction.

Summary: We have reported the first results of the numerical-relativity simulation performed incorporating both a finite-temperature (Shen's) EOS and neutrino cooling effect. We showed that for such a stiff EOS, HMNS is the canonical outcome and $\mathrm{BH}$ is not promptly formed after the onset of the merger as long as the total mass of the system is smaller than $3.2 M_{\odot}$. The primary reason is that thermal pressure plays an important role for sustaining the HMNS. We further showed that the lifetime of the formed HMNS with mass $\lesssim 3 M_{\odot}$ is much longer than its dynamical timescale, $\gg 10 \mathrm{~ms}$, and will be determined by the timescale of neutrino cooling. Neutrino luminosity of the HMNS was shown to be high as $\sim 3-10 \times 10^{53} \mathrm{erg} / \mathrm{s}$. The effective amplitude of GWs is $4-6 \times 10^{-22}$ at $f_{\text {peak }}=2.1-2.5 \mathrm{kHz}$ for a source distance of $100 \mathrm{Mpc}$. If the BNS merger happens at a relatively short source distance or is located in an optimistic direction, such GWs may be detected and HMNS formation will be confirmed.

Acknowledgments: Numerical simulations were performed on SR16000 at YITP of Kyoto University and on SX9 and XT4 at CfCA of NAOJ. This work was supported by Grant-in-Aid for Scientific Research (21018008, 21105511, 21340051, 22740178), by Grant-in-Aid for Scientific Research on Innovative Area (20105004), and HPCI Strategic Program of Japanese MEXT.
[1] J. Abadie, et al., Nucl. Instrum. Methods Phys. Res. Sect. A624, 223 (2010): T. Accadia, et al., Class. Quantum Grav. 28, 025005 (2011): K. Kuroda, et al., Class. Quantum Grav. 27, 084004 (2010).

[2] R. Narayan, B. Paczynski, and T. Piran, Astrophys. J. Lett. 395, L83 (1992): T. Piran, Rev. Mod. Phys. 76, 1143 (2005): E. Nakar, Phys. Rep. 442, 166 (2007).

[3] M. Shibata and K. Uryū, Phys. Rev. D 61, 064001 (2000).

[4] M. D. Duez, Class. Quantum Grav. 27, 114002 (2010).

[5] See, e.g., S. L. Shapiro and S. A. Teukolsky, Black Holes, White Dwarfs, and Neutron Stars, Wiley Interscience (New York, 1983).

[6] M. Shibata, K. Taniguchi, and K. Uryū, Phys. Rev. D 71, 084021 (2005); M. Shibata and K. Taniguchi, Phys. Rev. D 73, 064027 (2006).

[7] K. Kiuchi, et al., Phys. Rev. D 80, 064037 (2009).

[8] L. Rezzolla et al., Astrophys. J. Lett. 732, L6 (2011).
[9] M. Ruffert, H.-Th. Janka, and G. Schäfer, Astron. Astrophys. 311, 532 (1996); M. Ruffert and H.-Th. Janka, ibid 380, 544 (2001); S. Rosswog and M. Liebendörfer, Mon. Not. R. Astron. Soc. 342, 673 (2003).

[10] L. Dessart et al., Astrophys. J. 690, 1681 (2009).

[11] S. Setiawan, M. Ruffert, and H.-Th. Janka, Astron. Astrophys. 458, 553 (2006).

[12] R. Oechslin and H.-Th. Janka, Phys. Rev. Lett. 99, 121102 (2007); R. Oechslin, H.-Th. Janka, and A. Marek, Astron. Astrophys. 467, 395 (2007).

[13] H. Shen, et al., Nucl. Phys. A 637, 435 (1998); Prog. Theor. Phys. 100, 1013 (1998).

[14] Y. Sekiguchi, Prog. Theor. Phys. 124, 331 (2010).

[15] M. Shibata and T. Nakamura, Phys. Rev. D 52, 5428 (1995); T. W. Baumgarte and S. L. Shapiro, Phys. Rev. D 59, 024007 (1998); M. Campanelli, et al., Phys. Rev. Lett. 96, 111101 (2006). 
[16] P. Demorest, et al., Nature 467, 1081 (2010).

[17] I. H. Stairs, Science 304, 547 (2004); D. R. Lorimer, Living Review Relativity, 11, 8 (2008).

[18] K. Hotokezaka, et al., Phys. Rev. D, (2011) in press.
[19] E.g., K. Sumiyoshi, S. Yamada, and H. Suzuki, Astrophys. J. 629, 922 (2005).

[20] Y. Suwa and K. Murase, Phys. Rev. D 80, 123008 (2009). 